${ }^{1}$ К. С. Белюк, ${ }^{1}$ К. Н. Жандаров, ${ }^{1} Э$ Э. В. Могилевец, ${ }^{1}$ М. О. Радикевич,

${ }^{1}$ А. В. Заболотная, ${ }^{1}$ Р. С. Шило, ${ }^{2}$ О. С. Сорока

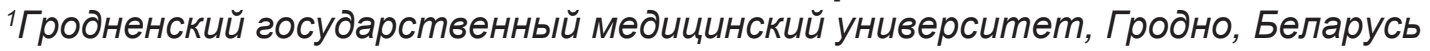

г Гродненская университетская клиника, Гродно, Республика Беларусь

Введение. Частота хронического панкреатита и его осложнений неуклонно растет, что ставит проблему лечения данной патологии в число актуальных и значимых.

Цель исследования - оптимизация хирургического лечения пациентов с гипертензионно-протоковым типом хронического панкреатита с протяженной стриктурой интрапанкреатической части общего желчного протока.

Материал и методы. В Гродненской университетской клинике за период 2006-202122., по поводу хронического панкреатита с патологией протоковой системы и в сочетании с кистами поджелудочной железы выполнено 328 операций. Общее количество осложнений составило - 5,1\%.

18 пациентам с хроническим рецидивирующим панкреатитом с протоковой гипертензией в сочетании с кистой головки поджелудочной железы, с протяженной стриктурой терминального отдела общего желчного протока и механической желтухой выполнены операции по разработанным в клинике методикам: 12 пациентам проведена холедохопанкреатоеюностомия, пятерым - холедохоцистопанкреатоеюностомия, холедохоцистодуоденостомия выполнена в одном случае.

Результаты. Разработанные методы, позволяют выполнить адекватное внутреннее дренирование протоковой системы поджелудочной железы и желчевыводящих путей, а также кистозных образований.

Выводы. Применение разработанных методов улучшает результаты хирургического лечения осложненного хронического панкреатита.

Ключевые слова: хронический панкреатит, механическая желтуха, стриктура терминального отдела холедоха, киста поджелудочной железы.

\title{
SURGICAL TREATMENT OF HYPERTENSION-DUCTAL TYPE OF CHRONIC PANCREATITIS, IN COMBINATION WITH BILIARY OBSTRUCTION SYNDROME
}

${ }^{1}$ K. S. Belyuk, ${ }^{1}$ K. N. Zhandarov, ${ }^{1}$ E. V. Mahiliavets, ${ }^{1}$ M. O. Radikevich, ${ }^{1}$ A.V. Zabolotnaya, ${ }^{1}$ R. S. Shyla, ${ }^{2}$ O. S. Soroka

${ }^{1}$ Grodno State Medical University, Grodno, Republic of Belarus

${ }^{2}$ Grodno University Clinic, Grodno, Republic of Belarus

Background. The frequency of chronic pancreatitis and its complications is steadily increasing, which makes the problem of treating this pathology one of the most urgent and significant.

Objective. Improvement of the results of surgical treatment of patients with hypertension-ductal type of chronic pancreatitis with extended stricture of the intrapancreatic part of the common bile duct.

Material and methods. During the period 2006-2021, 328 operations were performed in the Grodno University Clinic due to chronic pancreatitis with pathology of the ductal system in combination with pancreatic cysts. The total number of complications was $5,1 \%$.

18 patients with chronic recurrent pancreatitis, ductal hypertension in combination with a cyst of the head pancreas, also with extended stricture of the terminal part common bile duct and mechanical jaundice were operated on according to the methods developed in the clinic: 12 patients underwent choledochopancreatoejunostomy, 5 patients underwent choledochocystopancreatoejunostomy, choledochocystoduodenostomy was performed in 1 case.

Results. The developed methods make it possible to perform adequate internal drainage of the ductal system of the pancreas and bile ducts, as well as cystic formations.

Conclusions. The application of the developed methods improves the results of surgical treatment complicated chronic pancreatitis.

Keywords: chronic pancreatitis, mechanical jaundice, stricture of the terminal choledochus, pancreatic cyst.

Автор, ответственный за переписку:

Белюк Константин Сергеевич, канд. мед. наук, доц., Гродненский государственный медицинский университет;

e-mail: belyukks@ya.ru
Corresponding author:

Belyuk Kanstantsin, PhD (Medicine), Associate Professor; Grodno State Medical University; e-mail: belyukks@ya.ru 
Для цитирования: Хирургическое лечение гипертензи онно-протокового типа хронического панкреатита, в сочетании с синдромом билиарной обструкции / К. С. Белюк, К. Н. Жандаров, Э. В. Могилевец, М. О. Радикевич, А. В. Заболотная, Р. С. Шило, О. С. Сорока // Гепатология и гастроэнтерология. 2021. Т. 5, № 2. С. 174-183. https://doi.org/10.25298/2616-55462021-5-2-174-183
For citation: Belyuk KS, Zhandarov KN, Mahiliavets EV, Radikevich MO, Zabolotnaya AV, Shyla RS, Soroka OS. Surgical treatment of hypertension-ductal type of chronic pancreatitis, in combination with biliary obstruction syndrome. Hepatology and Gastroenterology. 2021;5(2):174-183. https://doi. org/10.25298/2616-5546-2021-5-2-174-183

\section{Введение}

Частота хронического панкреатита и его осложнений неуклонно растет, что ставит проблему лечения данной патологии в число актуальных и значимых [1].

Клиническая картина характеризуется постоянной или рецидивирующей интенсивной болью, а также разного рода осложнениями со стороны как самой железы, так и окружающих ее органов [1, 2]. Хронический панкреатит в 50-80\% случаев наблюдаются у лиц трудоспособного возраста, что придает проблеме его лечения не только медицинскую, но и социальную значимость [3, 4, 5]. Отмечается также неуклонный рост желчнокаменной болезни и ее осложнений, что является одной из причин развития хронического панкреатита $[6,7,8]$. В связи с ростом частоты осложненных форм желчнокаменной болезни и хронического панкреатита, а также анатомической общностью выводных протоков печени и поджелудочной железы наблюдается рост числа сочетанных заболеваний, а именно хронического панкреатита, осложненного протяженной стриктурой терминального отдела холедоха и механической желтухой. Поэтому возникает необходимость разработки наиболее эффрективных способов лечения сочетанной патологии.

В отношении хирургической тактики при кистах поджелудочной железы единой точки зрения нет. Характер, объем оперативного вмешательства зависят от этиологии, наличия или отсутствия связи их с протоковой системой, наличия осложнений. Применяются разные вмешательства: от чрескожных пункций, дренирования и склерозирования кист до резекции поджелудочной железы и панкреатодуоденальной резекции [2]. Несмотря на многообразие оперативных вмешательств при осложненных формах хронического панкреатита, результаты их не совсем удовлетворяют хирургов: радикальные операции в большинстве своем травматичны, дают высокий процент осложнений, а при паллиативных вмешательствах нередки рецидивы заболевания.

При хроническом панкреатите с протоковой гипертензией наиболее распространенные методы оперативного лечения - дренирующие операции, которые сводятся к созданию нового пути оттока панкреатического сока в разные отделы кишечника [3, 9, 10, 11]. Наиболее близкий к предлагаемым методам - способ продольной панкреатоеюностомии на выключенной из пищеварения петле тонкого кишечника по Ру, описанный М. В. Даниловым в 1995 г. [2]. Данный метод позволяет произвести дренирование только протоковой системы поджелудочной железы, что и является его недостатком.

Когда хронический рецидивирующий панкреатит с протоковой гипертензией сочетается с протяженной стриктурой терминального отдела общего желчного протока (ОЖП) и билиарной гипертензией, кистой головки поджелудочной железы, необходимо выполнить декомпрессию как протоковой системы поджелудочной железы (ПЖ), так и желчевыводящих путей. Появляется необходимость наложения двух анастомозов: панкреатоеюноанастомоза и одного из вариантов билиодигестивного анастомоза, что весьма затруднительно, удлиняет продолжительность операции и связано с разными осложнениями, характерными для 2 анастомозов. Поэтому возникает необходимость разработки новых способов хирургического лечения сочетанной патологии путем обеспечения возможности одновременного адекватного внутреннего дренирования общего желчного протока и главного панкреатического протока, кист поджелудочной железы, что и достигается с помощью предложенных нами методов.

Цель исследования - оптимизация хирургического лечения пациентов с гипертензионно-протоковым типом хронического панкреатита с протяженной стриктурой интрапанкреатической части общего желчного протока.

\section{Материал и методы}

В Гродненской университетской клинике за период 2006-2021 гг. по поводу хронического панкреатита с патологией протоковой системы и в сочетании с кистами поджелудочной железы выполнено 328 операций (табл. 1), из них: панкреатоеюностомия с холедохоеюностомией на одной петле кишки, отключенной по Ру - 23 операции, продольная панкреатоеюностомия по Ру выполнена в 56 случаях; панкреатоцистовирсунгоеюностомия по Ру в 70 случаях; дистальная резекция произведена 9 пациентам; гемипанкреатэктомия (дистальная) - 16 операций; клиновидная резекция головки и продольная клиновидная резекция, операция Izbicki в 79 случаях; срединная резекция - 8 операций; интрапаренхиматозная резекция головки - 45 пациентам; холедохопанкреатоеюностомия - 12 операций; 
холедохоцистопанкреатоеюностомия - 5 пациентам; холедохоцистодуоденостомия выполнена 1 пациенту; торакоскопическая спланхниксимпатэктомия - 4 операции.

Общее количество осложнений - 5,1\%.

Таблица 1. - Операции при сочетании кист поджелудочной железы с патологией протоков у пациентов с хроническим рецидивирующим панкреатитом.

Table 1. - Operations in combination of pancreatic cysts with duct pathology in patients with chronic recurrent pancreatitis.

\begin{tabular}{|c|c|c|}
\hline $\begin{array}{c}\text { Наименование } \\
\text { операции }\end{array}$ & $\begin{array}{c}\text { Кол-во } \\
\text { операций }\end{array}$ & $\begin{array}{c}\text { Кол-во } \\
\text { осложнений }\end{array}$ \\
\hline $\begin{array}{l}\text { Панкреатоеюностомия с } \\
\text { холедохоеюностоямией } \\
\text { на одной петле кишки, } \\
\text { отключённой по Ру }\end{array}$ & 23 & - \\
\hline $\begin{array}{l}\text { Продольная панкреатоею- } \\
\text { ностомия по Ру }\end{array}$ & 56 & $3(0,9 \%)$ \\
\hline $\begin{array}{l}\text { Панкреатоцистовирсунгоею- } \\
\text { ностомия по Ру }\end{array}$ & 70 & $5(1,5 \%)$ \\
\hline Дистальная резекция & 9 & - \\
\hline $\begin{array}{l}\text { Гемипанкреатэктомия } \\
\text { (дистальная) }\end{array}$ & 16 & $1(0,3 \%)$ \\
\hline $\begin{array}{l}\text { Клиновидная резекция } \\
\text { головки и продольная } \\
\text { клиновидная резекция, } \\
\text { операция Izbicki } \\
\end{array}$ & 79 & $4(1,2 \%)$ \\
\hline Срединная резекция & 8 & - \\
\hline $\begin{array}{l}\text { Интрапаренхиматозная } \\
\text { резекция головки }\end{array}$ & 45 & $3(0,9 \%)$ \\
\hline $\begin{array}{l}\text { Холедохопанкреатоеюносто- } \\
\text { мия }\end{array}$ & 12 & $1(0,3 \%)$ \\
\hline $\begin{array}{l}\text { Холедохоцистопанкреа- } \\
\text { тоеюностомия }\end{array}$ & 5 & - \\
\hline $\begin{array}{l}\text { Холедохоцистодуоде- } \\
\text { ностомия }\end{array}$ & 1 & - \\
\hline $\begin{array}{l}\text { Торакоскопическая } \\
\text { спланхниксимпатэктомия }\end{array}$ & 4 & - \\
\hline Итого: & 328 & $16(5,1 \%)$ \\
\hline
\end{tabular}

Наибольшую проблему хирургического лечения представляют пациенты, у которых хронический рецидивирующий панкреатит с протоковой гипертензией сочетается с протяженной стриктурой терминального отдела ОЖП и механической желтухой. Результаты оперативного лечения 18 таких пациентов представляются в данной работе.

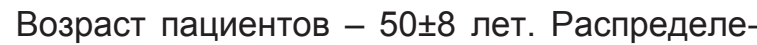
ние по полу: 16 мужчин, 2 женщины.

На дооперационном этапе всем пациентам проводились общеклинические методы исследования по стандартным методикам в клинической лаборатории Гродненской университетской клиники. Лабораторные исследования включали: полный развернутый анализ крови; общий анализ мочи с определением диастазы; биохимический анализ крови, включающий показатели билирубина, трансаминаз, щелочной фоссфатазы, амилазы крови, общего белка, глюкозы крови, мочевины; группу крови и коагулограмму.

Средний уровень общего билирубина на дооперационном этапе составил 40 [16; 128] мкмоль/л, амилазы - 209 [74; 473] Ед/л, АСТ 85 [36; 157] Ед, АЛТ - 75 [40; 179] Ед (табл. 2).

Таблица 2. - Результаты основных показателей БАК

Table 2. - Results of the main indicators of blood biochemistry

\begin{tabular}{|l|c|c|c|}
\hline \multicolumn{1}{|c|}{ Показатели } & $\begin{array}{c}\text { Медиана и } \\
\text { квартили }\end{array}$ & Max & Min \\
\hline $\begin{array}{l}\text { Билирубин } \\
\text { (мкмоль/л) }\end{array}$ & $40[16 ; 128]$ & 187 & 28 \\
\hline Амилаза (Ед/л) & $209[74 ; 473]$ & 1026 & 48 \\
\hline АСТ (Ед) & $85[36 ; 157]$ & 623 & 28 \\
\hline АЛТ (Ед) & $75[40 ; 179]$ & 814 & 16 \\
\hline
\end{tabular}

Наряду с лабораторными исследованиями выполнялись инструментальные методы диагностики. Всем пациентам на дооперационном этапе ыло выполнены УЗИ и МРТ органов брюшной полости (рис. 1).
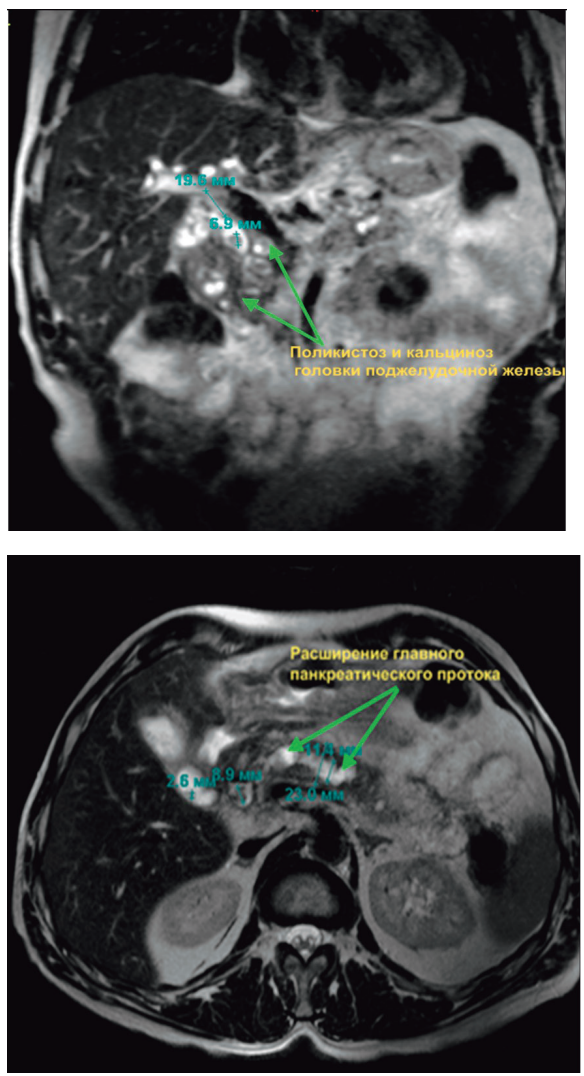

Рисунок1(а,б).-МРТ-грамма:поликистозикальцинозголовки поджелудочной железы с внутрипротоковой гипертензией Figure $1(a, b)$. - MRl-gram: polycystic and calcinous head of the pancreas with intra-flow hypertension 
По данным МРТ, средний размер головки

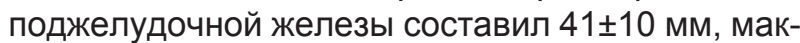
симальный размер - 61 мм, минимальный - 27 мм. Расширение главного панкреатического про-

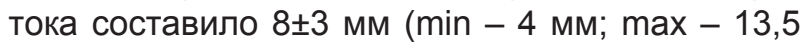
мм). У 12 (66,7\%) пациентов наряду с панкреатической гипертензией установлено наличие кистозных образований головки поджелудочной железы. Кроме того, у всех пациентов выявлено наличие протяженной стриктуры интрапанкреатической части холедоха с престенотическим его расширением до 17士5 мм (табл. 3).

Таблица 3. - Результаты исследования МРТ Table 3. - MRI results

\begin{tabular}{|c|c|c|c|}
\hline Показатели & $\begin{array}{c}\text { Среднее } \\
\text { стандартное } \\
\text { отклонение } \\
\text { (мм) }\end{array}$ & $\begin{array}{l}\text { Max } \\
\text { (MM) }\end{array}$ & Min (Mм) \\
\hline $\begin{array}{l}\text { Размеры } \\
\text { головки ПЖ }\end{array}$ & $41 \pm 10$ & 61 & 27 \\
\hline $\begin{array}{l}\text { Размеры тела } \\
\text { ПЖ }\end{array}$ & $23 \pm 6$ & 33 & 12 \\
\hline $\begin{array}{l}\text { Размеры хвоста } \\
\text { Пж }\end{array}$ & $19 \pm 5$ & 26,5 & 10 \\
\hline $\begin{array}{l}\text { Расширение } \\
\text { главного пан- } \\
\text { креатического } \\
\text { протока }\end{array}$ & $8 \pm 3$ & 13,5 & 4 \\
\hline $\begin{array}{l}\text { Киста головки } \\
\text { ПЖ }\end{array}$ & \multicolumn{3}{|c|}{ У 66,7 \% пациентов } \\
\hline Диаметр ОЖП & \multicolumn{3}{|c|}{$17 \pm 5$ мм } \\
\hline $\begin{array}{l}\text { Расширение } \\
\text { ВПЖП }\end{array}$ & \multicolumn{3}{|c|}{$100 \%$ у всех } \\
\hline
\end{tabular}

Одному пациенту из-за выраженной механической желтухи на первом этапе была наложена разгрузочная холецистостома и выполнена холангиография (рис. 2), на которой отмечалось расширение внутрипечёночных желчных протоков и ОЖП. Диагностирована стриктура ин-

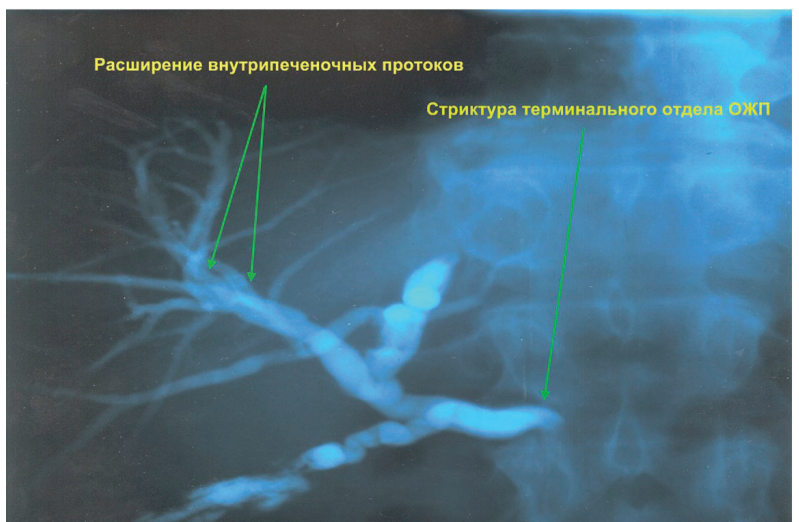

Рисунок 2. - Холангиограмма: стриктура терминального отдела холедоха с билиарной гипертензией

Figure 2. - Cholangiogram: stricture of the terminal part of the choledochus with biliary hypertension трапанкреатической части холедоха, контраст в двенадцатиперстную кишку не поступал.

Проводились инструментальные методы исследования, которые включали: рентгенографрию органов грудной клетки, фрлюорографию, электрокардиографию, выполнялась ФГДС.

12 пациентам с хроническим рецидивирующим панкреатитом с протоковой гипертензией в сочетании с протяженной стриктурой терминального отдела ОЖП и билиарной гипертензией выполнено одномоментное внутреннее дренирование холедоха и протоковой системы поджелудочной железы (патент РБ № 15800 от 16.06.2009; инструкция по применению № 0280411 от 18.11.2011).

Как видно на МРТ-грамме (рис. 3), у данных пациентов отмечалось увеличение размеров головки поджелудочной железы с кальцинозом и поликистозом, расширение Вирсунгова протока и ОЖП с протяженной стриктурой в интрапанкреатической части.
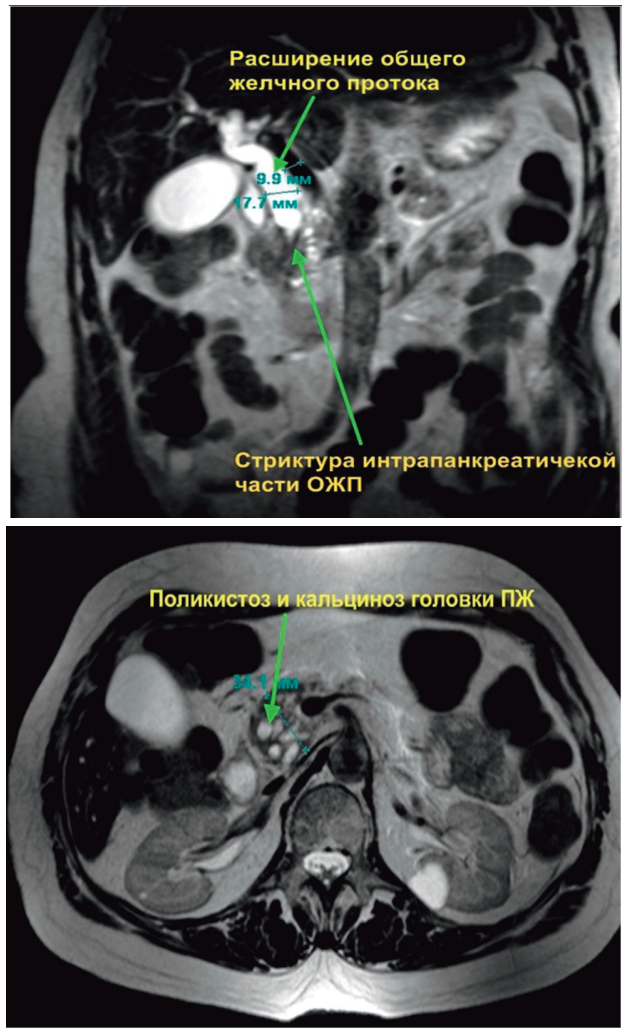

Рисунок 3 (а,б). - МРТ-грамма: поликистоз и кальциноз головки ПЖ в сочетании с панкреатической и билиарной гипертензией

Figure $3(a, b)$. - MRI grams: polycystic and calcified pancreatic head combined with pancreatic and biliary hypertension

Холедохопанкреатоеюностомия выполнялась по следующей методике (рис. 4):

Под общим обезболиванием в положении пациента на спине с подложенным под спину валиком в области X-XI ребер выполняют верхнесрединную лапаротомию. Доступ к поджелудочной железе осуществляют через желудочно-ободоч- 
ную связку. Выполняется субтотальная клиновидная резекция головки поджелудочной железы, продольная вирсунготомия длиной до 6-12 см и клиновидная резекция тела и хвоста поджелудочной железы.

Вторым этапом производится холецистэктомия. Через культю пузырного протока или холедохотомическое отверстие выполняется ревизия желчевыводящих протоков посредством зондирования, интраоперационной холангиографии или фииброхоледохоскопии для установления уровня начала стеноза интрапанкреатической части ОЖП.

В последующем, на металлическом зонде Долиотти, введенном через культю пузырного протока или холедохотомическое отверстие, в престенотической части ОЖП выполняется с помощью диатермокоагулятора клиновидное иссечение ткани головки поджелудочной железы до стенки общего желчного протока, в который введен металлический зонд. На этом металлическом зонде и проводится вскрытие ОЖП с продлением разреза вверх до 1,0-1,5 см для формирования адекватного оттока желчи. При необходимости накладываются швы из рассасывающегося материала (дексон 310) на атравматической игле для герметизации наложенного холедохопанкреатического сообщения от забрюшинной клетчатки.

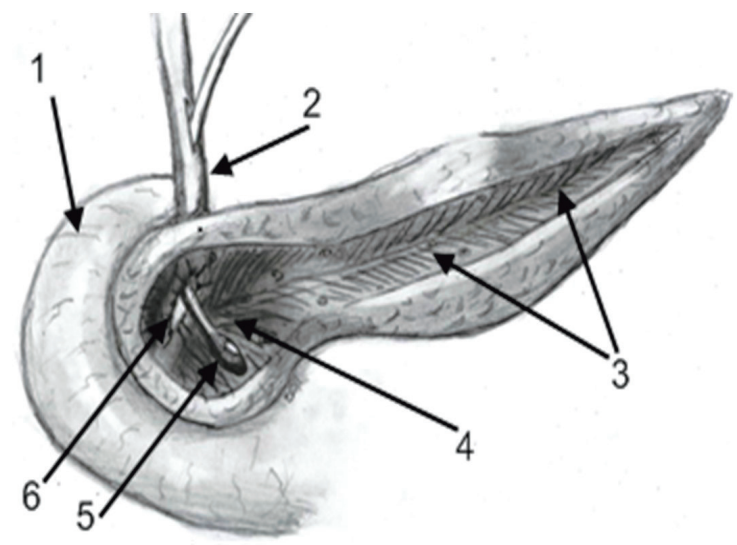

Рисунок 4. - Формирование холедохопанкреатического сообщения. 1.Двенадцатиперстная кишка. 2.Общий желчный проток. З.Клиновидно резецированное тело и хвост поджелудочной железы. 4. Клиновидно резецированная головка поджелудочной железы. 5. Зонд Долиотти. 6. Холедохопанкреатическое сообщения

Figure 4. - Formation of the choledochopancreatic compound.

1.Duodenum. 2. Common bile duct. 3. Sphenoid resected body and tail of the pancreas. 4.Sphenoid resected head of the pancreas. 5.Doliotti probe. 6.Choledochopancreatic compound.

В последующем через культю пузырного протока вводится дренаж (типа Холстеда-Пиковского) и выводится через сформированное нами холедохопанкреатическое соустье во вскрытый панкреатический проток, при этом отверстия в дренаже располагаются как в просвете ОЖП, так и выходят в просвет панкреатического протока. Фиксируется дренаж наложением лигатуры из рассасывающегося шовного материала (короткого срока) на культю пузырного протока. После контроля гемостаза приступают к следующему этапу операции - наложению изоперистальтического продольного панкреатоеюноанастомоза на выключенной из пищеварения по методике РУ петле тощей кишки длиной 30-40 см. Брюшную полость ушивают наглухо, дренируют несколькими дренажами - по правому и левому боковым каналам и малый таз.

Использование холедохопанкреатоеюностомии позволяет адекватно выполнить внутренне дренирование как ОЖП, так и протоковой системы поджелудочной железы.

Пяти пациентам, страдающим гипертензионно-протоковым типом хронического панкреатита, осложненного кистой головки поджелудочной железы и протяженной стриктурой терминального отдела холедоха с механической желтухой, выполнена холедохоцистопанкеатоеюностомия. В данной ситуации необходимо выполнить внутреннее дренирование кисты ПЖ, панкреатической и билиарной системы.

На представленной МРТ-грамме (рис. 5) отмечается расширение ОЖП до 18,4 мм со стриктурой в терминальном отделе, где определяется киста головки поджелудочной железы. Имеет место также расширение Вирсунгового протока до 8 мм. В данной ситуации необходимо выполнить внутреннее дренирование кисты ПЖ, панкреатической и билиарной системы.

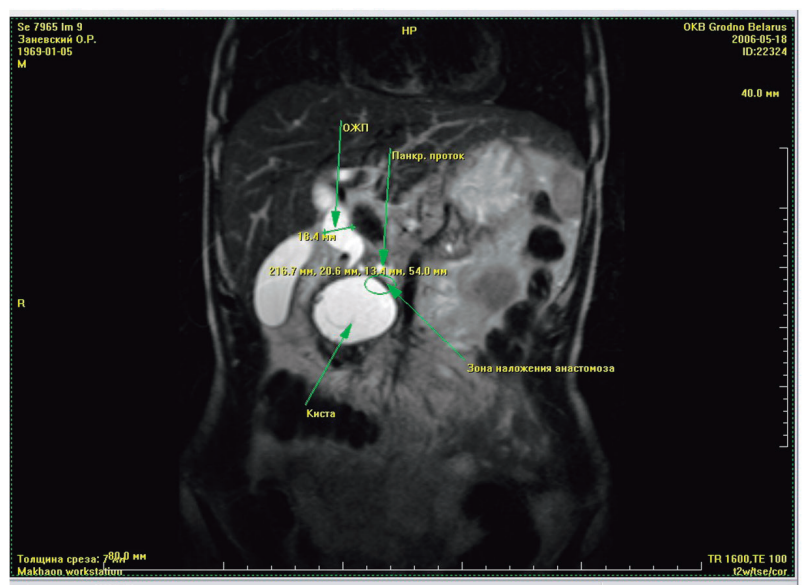

Рисунок 5. - МРТ-грамма: киста головки поджелудочной железы с протяженной стриктурой терминального отдела холедоха и расширением Вирсунгова протока. Figure 5. - MRI-gram: a cyst of the head pancreas with an extended stricture of the terminal part choledochus and an extension of the Virsung duct.

По разработанной в клинике методике данным пациентам выполнена холедохоцистопанкреатоеюностомия (рис. 6). Под общим обезболиванием выполняют верхнесрединную лапаротомию. Доступ к поджелудочной железе осуществляют через желудочно-ободочную связку. После пункции кисты головки поджелудочной железы производят ее вскрытие и иссечение передней 
стенки с субтотальной интрапаренхиматозной резекцией головки поджелудочной железы и продольной клиновидной резекцией тела и хвоста железы. Далее производят холецистэктомию. В последующем, на металлическом зонде Долиотти, введенном через культю пузырного протока, проводится вскрытие ОЖП в просвет кисты головки поджелудочной железы длиной 1,0-1,5 см для формирования адекватного оттока желчи. Накладываются швы из рассасывающегося материала (дексон 310) на атравматической игле для герметизации наложенного холедохоцистопанкреатического сообщения от забрюшинной клетчатки.

В последующем через культю пузырного протока вводится дренаж (типа Холстеда-Пиковского) и выводится через сформированное нами холедохоцистопанкреатическое соустье, при этом отверстия в дренаже располагаются как в просвете ОЖП, так и выходят в просвет кисты поджелудочной железы и полости, образовавшейся в результате интрапаренхиматозной резекции головки поджелудочной железы и продольной клиновидной резекции тела и хвоста поджелудочной железы. Фиксируется дренаж наложением лигатуры из рассасывающегося шовного материала на культю пузырного протока. После контроля гемостаза приступают к следующему этапу операции - наложению изоперистальтического продольного панкреатоцистоеюноанасто-

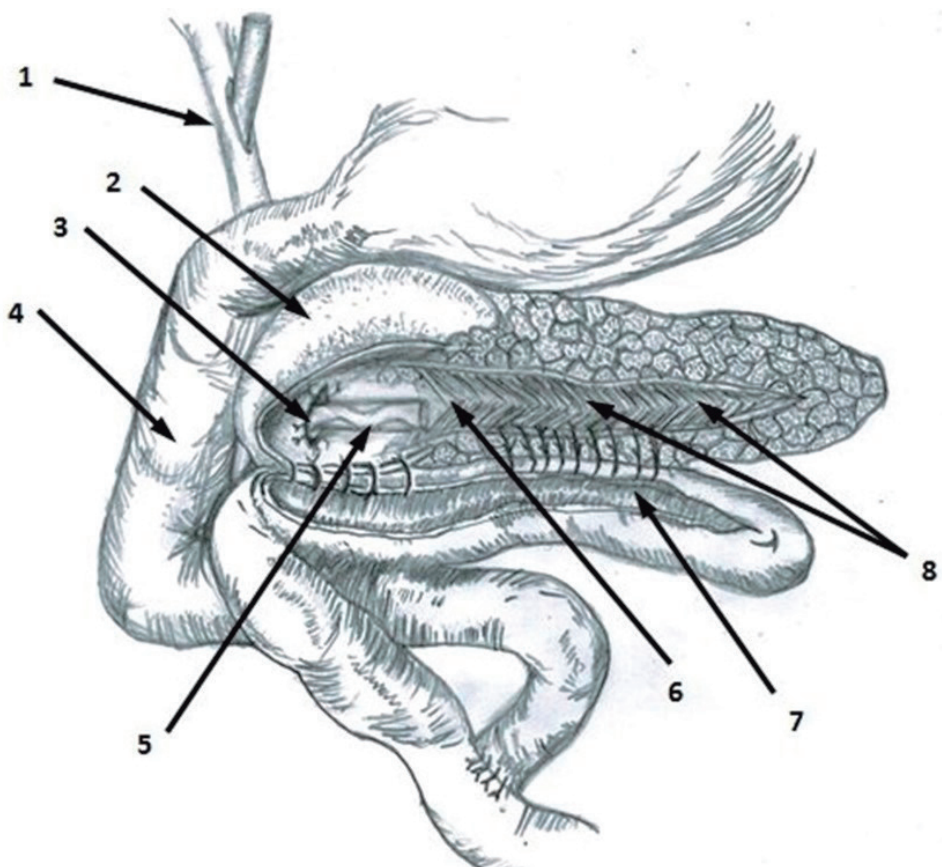

Рисунок 6. - Холедохоцистопанкреатоеюностомия. 1.Общий желчный проток. 2.Киста головки поджелудочной железы. З.Холедохоцистопанкреатическое сообщение. 4.Двенадиатиперстная кишка. 5.Дренажная трубка. 6.Субтотально резецированная головка поджелудочной железы. 7.Выключенная по Ру петля кишки. 8.Клиновидно резецированное тело и хвост поджелудочной железы. Figure 6. - Choledochocystopancreatoejunostomy. .Common bile duct. 2.Cyst of the head pancreas. 3.Choledochocystopancreatic compound. 4.Duodenum. 5.Drainage tube. 6. Subtotally resected head of the pancreas. 7. The loop of the intestine turned off (by Ru). 8.Sphenoid resected body and tail of the pancreas. моза на выключенной из пищеварения по методике РУ петле тощей кишки длиной 30-40 см.

Осуществление предложенного метода подтверждается следующим примером конкретного использования в клинике.

Пациент «К», 1950 г. р., поступил в отделение хирургической панкреатологии, гепатологии и трансплантации органов и тканей Гродненской бластной клинической больницы 26.01.2015.

Анамнез заболевания: 18.01.2015 госпитализирован в хирургическое отделение Слонимцой ЦРБ с клиникой механической желтухи 2.2014 пациенту выполнена холецистэктод поводу желчного перитонита и дренирование холедоха по Вишневскому. За время лечения в состояние пациента - без положительной

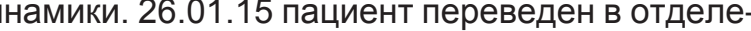
трансплантации органов и тканей.

Пациент обследован:

ФГДС с осмотром большого дуоденального сосочка: Фатеров сосочек правильной формы, на момент осмотра оттока желчи не отмечается.

МРТ брюшной полости: в передней стенки живота имеется зона неоднородности, характерная для послеоперационных осложнений. Внутрипечёночные желчные протоки не расширены, холедох до 7 мм в диаметре. Поджелудочная железа обычно расположена, с нечеткими неровными контурами, сигнал от паренхимы неоднородный, в основном умеренно повышенный, в задней левой части головки имеется округлой формы жидкостный компонент около 11,5 мм диаметром, помимо того, в передней части головки - округлой формы участок неоднородной паренхимы до 16,5 мм диаметром. Вирсунгов проток расширен до 7,5 мм, заканчивается в области головки. Парапанкреатическая клетчатка не изменена. Спереди от головки имеется однородный тонкостенный жидкостный компонент $48,5 \times 39,5 \times 54$ мм.

Клинический диагноз: Хронический рецидивирующий панкреатит, гипертензионно-протоковый тип. Киста головки поджелудочной железы. Протяженный стеноз интрапанкреатической части общего желчного протока, механическая желтуха, холангит. Полный наружный желчный свищ.

03.02.2015 - операция: повторная лапаротомия, висцеролиз. Клиновидная субтотальная резекция головки поджелудочной железы, холедохо- 
цистопанкреатоеюностомия по Ру. Дренирование холедоха и зоны холедохоцистопанкреатоеюноанастомоза по типу Холстеда-Пиковского. Дренирование брюшной полости.

На операции: повторная лапаротомия, висцеролиз, дренаж Вишневского установлен под культей пузырного протока, где вскрыт желчный подпечёночный абсцесс $3 \times 4$ см, гепатодуоденальная связка выделена из массивных рубцовых тканей. Общий желчный проток до 1,2 см шириной. Выделена культя пузырного протока, из которой под давлением поступает желчь. При фиброхоледохоскопии установлено наличие протяженной стриктуры в терминальном интрапанкреатическом отделе общего желчного протока. Поджелудочная железа с признаками воспаления и псевдотуморзного панкреатита. В области головки поджелудочной железы киста $4 \times 5$ см. Вскрыт главный панкреатический проток до 0,7 см в диаметре. Вскрыта (с частичным иссечением стенок) киста в области головки поджелудочной железы и соединена с главным панкреатическим протоком. Выполнена клиновидная субтотальная резекция головки поджелудочной железы. Учитывая наличие протяженной стриктуры общего желчного протока в интрапанкреатическом отделе, на зонде произведено рассечение стенки кисты поджелудочной железы и стенки ОЖП на протяжении 1,5 см с формированием холедохоцистопанкреатического анастомоза, получен отток желчи, рассечение произведено с помощью диатермокоагулятора. Таким образом, сформирована единая полость, в которую открываются киста ПЖ, главный панкреатический проток и общий желчный проток. Через культю пузырного протока и сфрормированное холедохоцистопанкреатическое соустье проведен дренаж по типу ХолстедаПиковского. Наложен продольный панкреатоцистоеюноанастомоз на выключенной по Ру петлей тощей кишки бок в бок. Дренирование брюшной полости, послойный шов раны.

Послеоперационный период протекал без осложнений.
27.02.2015 пациент выписан в удовлетворительном состоянии под наблюдение хирурга по месту жительства.

Таким образом, использование данного метода хирургического лечения позволяет одномоментно выполнить внутреннее дренирование ОЖП, кисты головки поджелудочной железы и панкреатической системы.

Пациенту, 49-ти лет, длительно страдающему хроническим рецидивирующим панкреатитом, осложненным кистой головки поджелудочной железы с хронической дуоденальной непроходимостью, протяженной стриктурой терминального отдела холедоха, с механической желтухой выполнена холедохоцистодуоденостомия. При обследовании по данным УЗИ и МРТ органов брюшной полости установлено: желчный пузырь $130 \times 50$ мм с застойным содержимым, ОЖП 12 мм диаметром, прослеживается до уровня головки поджелудочной железы, где определяется жидкостное образование 90×65 мм с плотной стенкой. Расширения главного панкреатического протока у данного пациента не выявлено, его диаметр составил 3 мм.

По разработанной в клинике методике пациенту выполнено хирургическое вмешательство (рис. 7): лапаротомия, холецистэктомия, дуоденотомия. Через заднюю стенку 12-перстной кишки вскрыта киста головки поджелудочной железы (по «типу» Юраша), сформирован цистодуоденоанастомоз. Интраоперационно с помощью

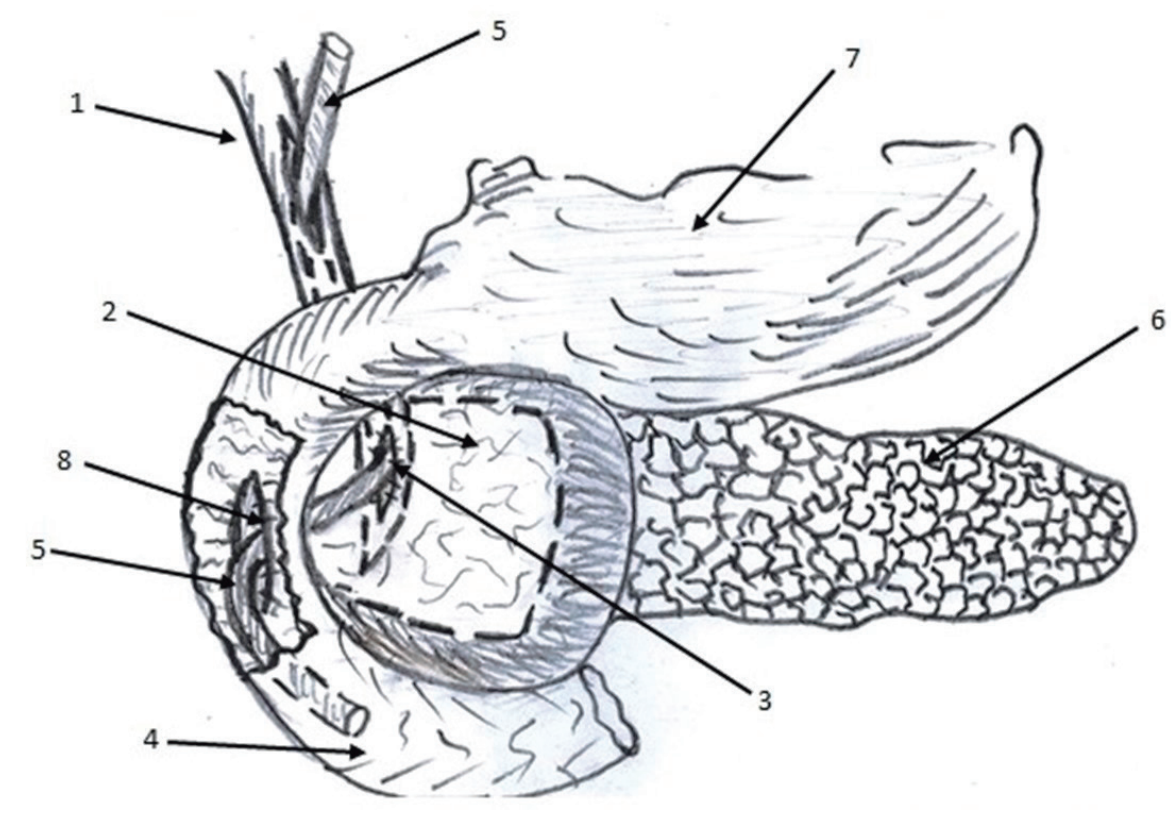

Рисунок 7. - Холедохоцистодуоденоностомия 1. Общий желчный проток. 2. Киста головки поджелудочной железы. 3. Холедохоцистоанастомоз.

4. Двенадцатиперстная кишка. 5.Дренажная трубка. 6.Поджелудочная железа. 7. Желудок. 8. Дуаденоцистоанастомоз (по «типу» Юраша).

Figure 7. - Choledochocystoduodenostomy. 1. Common bile duct.

2. Cyst of the head pancreas. 3. Choledochocystoanastomosis. 4. Duodenum. 5. Drainage tube. 6.Pancreas. 7.Stomach.

8. Duadenocystoanastomosis (according to the" type " of Jurash). 
зондирования ОЖП и фриброхоледохоскопии подтверждено наличие протяженной стриктуры терминального отдела холедоха, вовлеченного в склеротический процесс в области кисты головки поджелудочной железы. На металлическом буже Долиотти, введенном в культю пузырного протока, через сформированный цистодуоденоанастомоз выполнена холедохоцистостомия (длиной около 1,5 cм) с наложением швов между общим желчным протоком и стенкой кисты поджелудочной железы монофиламентной рассасывающейся нитью 4/0. Холедох и сформированный холедохоцистодуоденоанастомоз через пузырный проток дренирован до нижнегоризонтальной ветви 12-перстной кишки, последняя ушита двурядным швом.

Таким образом, было выполнено одновременное внутренне дренирование ОЖП и кисты головки поджелудочной железы через заднюю стенку двенадцатиперстной кишки.

Результаты и обсуждение

Во время операций осложнений не было. В раннем послеоперационном периоде осложнение в виде микронесостоятельности панкреатоеюноанастомоза, купировавшегося консервативными мероприятиями (благодаря его дренированию), отмечено у 1 пациента с холедохопанкреатоеюностомией. У остальных пациентов осложнений в раннем послеоперационном периоде не выявлено.

В послеоперационном периоде для изучения и контроля проходимости наложенных анасто-

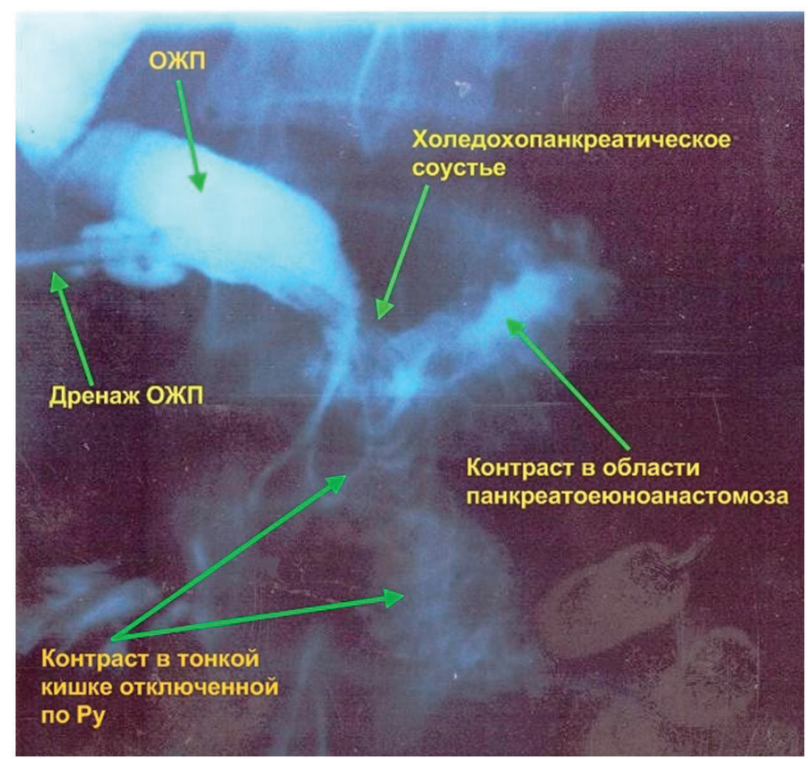

Рисунок 8. - Фистулография (холедохопанкреатоеюностомия).

Figure 8. - Fistulography (choledochopancreatoejunostomy)

мозов пациентам выполнялась фистулография (рис. 8).

Как видно на рентгенограмме, контрастное вещество заполняет ОЖП, через холедохопанкреатическое сообщение попадает в продольный панкреатоеюноанастомоз и далее - в тощую кишку, отключенную по Ру.

Для обоснования адекватности дренирования панкреатической и желчевыводящей систе-

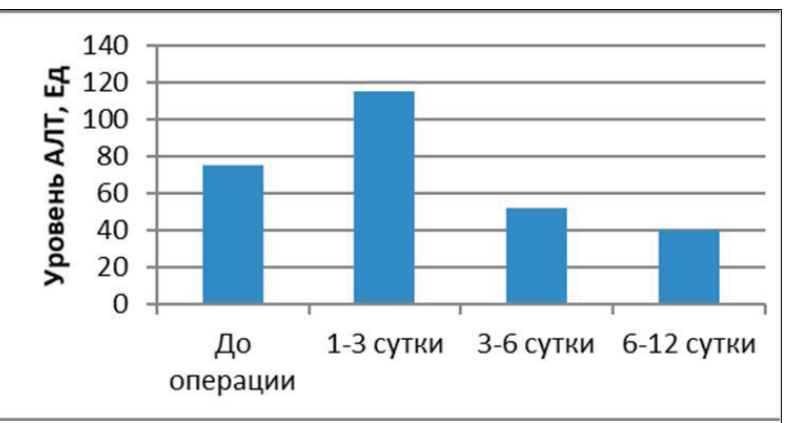

Рисунок 9. -Динамика изменения уровня амилазы и общего билирубина крови.

Figure 9. - Dynamics of changes in the level of amylase and total bilirubin in the blood.
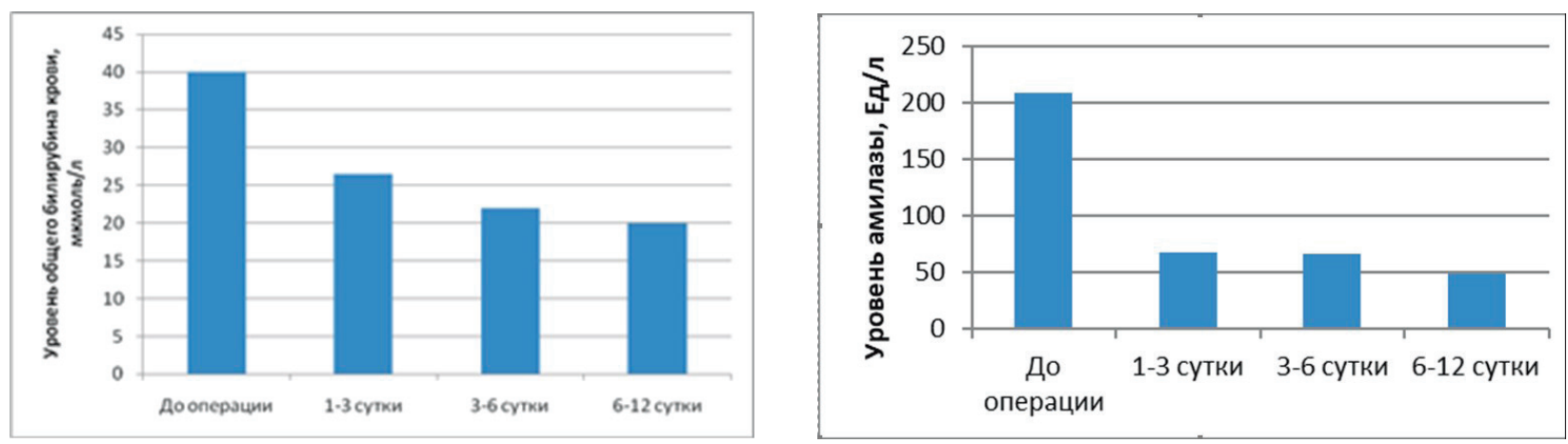

Рисунок 10. -Динамика изменения уровня АСТ и АЛТ.

Figure 10. - Dynamics of changes in AST and ALT levels. 
мы с помощью разработанных методик проанализировали также динамику снижения уровня общего билирубина, амилазы крови, печёночных фрерментов в послеоперационном периоде на 1-3, 3-6, 6-12 сутки (рис. 9, 10).

Как видно из рисунка 9, отмечалось статистически достоверное (критерий Уилкоксона, $p<0,05)$ снижение уровня общего билирубина и амилазы крови в послеоперационном периоде, что свидетельствует об адекватном внутреннем дренировании билиарной и панкреатической систем.

Снижение уровня АЛТ, АСТ (рис. 10) в послеоперационном периоде указывает на восстановление функций печени.

Удаление наружного дренажа проводилось в среднем на 16-18 сутки в послеоперационном периоде. Среднее количество койко-дней соста-

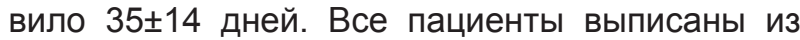
клиники в удовлетворительном состоянии.

В позднем послеоперационном периоде (через 4 года после оперативного вмешательства) осложнение в виде рецидива механической желтухи из-за прогрессирования склеротического процесса и стеноза холедопанкреатического сообщения отмечено у одного пациента, которому при повторном поступлении был наложен холедохоеюноананстомоз на имеющейся петле тонкой кишки, отключенной по Ру.

У остальных пациентов рецидива болевого синдрома и желтухи не выявлено при сроках наблюдения от 1 до 5 лет.

Таким образом, при сочетанной патологии, когда хронический рецидивирующий панкреатит с протоковой гипертензией, с кистой головки поджелудочной железы сочетается с протяженной стриктурой терминального отдела общего желчного протока и билиарной гипертензией, необходимо выполнить декомпрессию как протоковой системы и кисты Пж, так и желчевыводящих путей, что и достигается с помощью разработанных методов.

Предложенные методы имеют ряд преимуществ:

1. Не требуется 2 изолированные петли тонкой кишки для формирования раздельных панкреатоцистоеюноанастомоза и холедохоеюноанастомоза или холедоходуоденоанастомоза.

2. Осуществляется декомпрессия как протоковой системы и кистозных образований поджелудочной железы, так и желчевыводящих протоков.

3. Уменьшается время оперативного вмешательства за счет более быстрого наложения холедохопанкреатического сообщения или холедохоцистоанастомоза.

4. Данные способы могут быть осуществлены при холангите с инфильтративно-воспалительными изменениями стенки общего желчного протока и при любом его диаметре.

5. Осуществляется декомпрессия сформированных анастомозов через наружный дренаж.

\section{Выводы}

При использовании данных способов хирургического лечения осложнений хронического панкреатита осуществляется адекватная декомпрессия кистозных образований, протоковой системы поджелудочной железы и желчевыводящих путей. Не требуется формирования раздельных панкреатоцистоеюноанастомоза и холедохоеюноанастомоза или холедоходуоденоанастомоза, что уменьшает продолжительность операции и количество послеоперационных осложнений. Методы не требуют сверхвысокоточного оборудования, доступны по выполнению хирургами, имеющими достаточную квалификацию, могут быть с успехом применены во многих организациях здравоохранения Республики Беларусь.

\section{References}

1. Alperovich BI, Chaj VF, Habas GN. Lechenie kist i svishhe podzheludochnoj zhelezy. Annaly hirurgicheskoj gepatologii [Annals of HPB Surgery]. 2000;5(1):70-76. (Russian).

2. Danilov MV, Fedorov VD. Hirurgija podzheludochnoj zhelezy. Moskva: Medicina; 1995. 512 p. (Russian).

3. Kudrjashova IV. Rannjaja diagnostika hronicheskogo pankreatita s pozicii ispolzovanija ultrazvukovoj tomografii indeksa kachestva zhizni. Smolensk; 2003. 23 p. (Russian).

4. Sazhin VP, Avdovenko AL, Jurishhev VA. Principy differencirovannogo lechenija ostrogo pankreatita. Vestnik hirurgii im. I.I. Grekova [Grekov's bulletin of surgery]. 2004;163(1):56-59. (Russian).

5. Mori T, Abe N, Sugiyama M, Atomi Y, Way LW. Laparoscopic pancreatic cystgastrostomy. J Hepatobiliary Pancreat Surg. 2000;7(1):28-34. doi: 10.1007/s005340050150.

6. Galperin Jel, Vetshev PS. Rukovodstvo po hirurgii zhelchnyh putej. Moskva: Vidar; 2006. 568 p. (Russian).

7. Filippov SI, Nizovoj KA, Maljuk AI, Kocovskij MI, Kocovskij AM. Analiz rezultatov lechenija oslozhnennyh form ostrogo holecistita. In: Materialy XIII Mezhdunarodnoj konferenci hirurgov-gepatologov Rossii i stran SNG; 2006 Sep. 27-29;
Almaty: Annaly hirurgicheskoj gepatologii; 2006. p. 122. (Annals of HPB Surgery; vol. 11, no. 1.). (Russian).

8. Hadzhibaev AM, Atadzhanov ShK, Hoshimov MM. Jendoskopicheskaja hirurgija kalkuleznogo holecistita v sochetanii $s$ dobrokachestvennymi porazhenijami vnepechenochnyh zhelchnyh protokov [Endoscopic surgery for calculous cholecystitis in combination with nonmalignant affection of extra-hepatic bilious ducts]. Hirurgija. Zhurnal im. N.I. Pirogova [Pirogov Russian Journal of Surgery]. 2009;(2):40-43. (Russian).

9. Pitchumoni CS. Pathogenesis and managenent of pain in chronic pancreatitis. World J Gastroenterol. 2000;6(4):490496. doi: 10.3748/wjg.v6.i4.490.

10. Prinz RA, Greenlee HB. Pancreatic duct drainage in 100 patients with chronic pancreatitis. Ann Surg. 1981;194(3):313-320. doi: 10.1097/00000658-19810900000009 .

11. Sakorafas GH, Sarr MG. Changing trends in operations for chronic pancreatitis: a 22-year experience. Eur J Surg. 2000;166(8):633-637. doi: 10.1080/110241500750008295. 
Конфликт интересов. Авторы заявляют об отсутствии конфрликта интересов.

Финансирование. Исследование проведено без спонсорской поддержки.

Соответствие принципам этики. Исследование одобрено локальным этическим комитетом.

Сведения об авторах:

Белюк Константин Сергеевич, канд. мед. наук, доц., УО «Гродненский государственный медицинский университет»; e-mail: belyukks@ya.ru, ORCID: 0000-0001-8861-6835

Жандаров Константин Николаевич, д-р мед. наук, проф., УО «Гродненский государственный медицинский университет»; e-mail: zhandarov56@mail.ru

Могилевец Эдуард Владиславович, канд. мед. наук, доц. УО «Гродненский государственный медицинский университет»; e-mail: emogilevec@yandex.ru, ORCID: 0000-0001-75420980

Шило Руслан Сергеевич, УО «Гродненский государственный медицинский университет»; e-mail: shilo.ruslan@yandex. ru, ORCID: 0000-0003-0077-181X

Радикевич Михаил Олегович, УО «Гродненский государственный медицинский университет»; e-mail: miharadikevich@ gmail.com

Заболотная Алина Владимировна, УО «Гродненский государственный медицинский университет»; e-mail alina.zabolotnaya.00@mail.ru

Сорока Олег Станиславович, Гродненская университетская клиника, e-mail: regclinic@mail.grodno.by
Conflict of interest. The authors declare no conflict of interest.

Financing. The study was performed without external funding.

Conformity with the principles of ethics. The study was approved by the local ethics committee.

Information about authors:

Belyuk Kanstantsin, PhD (Medicine), Associate Professor, Grodno State Medical University, e-mail: belyukks@ya.ru ORCID: 0000-0001-8861-6835

Zhandarov Konstantin, PhD, MD (Medicine), Professor Grodno State Medical University, e-mail: zhandarov56@mail.ru

Mahiliavets Eduard, PhD (Medicine), Associate Professor, Grodno State Medical University, e-mail: emogilevec@yandex. ru, ORCID: 0000-0001-7542-0980

Shyla Ruslan, Grodno State Medical University, e-mail: shilo.ruslan@yandex.ru, ORCID: 0000-0003-0077-181X

Radikevich Mikhail, Grodno State Medical University, e-mail: miharadikevich@gmail.com

Zabolotnaya Alina, Grodno State Medical University, e-mail: alina.zabolotnaya.00@mail.ru;

Soroka Oleg, Grodno State Medical University, e-mail: regclinic@mail.grodno.by;
Поступила: 14.06.2021

Принята к печати: 14.10.2021
Received: 14.06.2021

Accepted: 14.10.2021 